\title{
10 years since TORCH: shining a new light on the risks of inhaled corticosteroids in COPD
}

\author{
James D. Chalmers and Holly R. Keir \\ Affiliation: Scottish Centre for Respiratory Research, University of Dundee, Ninewells Hospital and Medical \\ School, Dundee, UK. \\ Correspondence: James D. Chalmers, Division of Molecular and Clinical Medicine, University of Dundee, \\ Dundee, DD1 9SY, UK. E-mail: jchalmersadundee.ac.uk
}

@ERSpublications

New research identifies an increased risk of NTM infection in inhaled steroid users http://ow.ly/Sk8130ej5Oa

Cite this article as: Chalmers JD, Keir HR. 10 years since TORCH: shining a new light on the risks of inhaled corticosteroids in COPD. Eur Respir J 2017; 50: 1701582 [https://doi.org/10.1183/13993003.015822017].

10 years ago, in February 2007, the Towards a Revolution in COPD Health (TORCH) study was published in the New England Journal of Medicine [1]. While the objective of the study was to demonstrate a survival benefit of inhaled corticosteroids (ICS) in combination with long-acting beta-agonists (LABA) compared to placebo in chronic obstructive pulmonary disease (COPD), arguably the most lasting impact of the study on the field of COPD has been a progressive re-evaluation of the safety of ICS [1].

The study of 6184 patients failed to demonstrate a significant survival benefit $(p=0.052)$. There was, however, a significant increase in the frequency of pneumonia in patients with combined ICS/LABA ( $19.6 \%$ over 3 years) compared with $12.3 \%$ in the placebo group, $13.3 \%$ in the salmeterol group and $18.3 \%$ in the group receiving fluticasone alone [1]. While the TORCH study was by no means the first study to report pneumonia in association with ICS use, the findings of TORCH and subsequent analyses confirming the association between ICS and pneumonia have had a striking impact on the field [2]. Later trials looked more carefully, with routine use of chest radiography, for pneumonia as a specific event [3-5]. All ICS preparations have subsequently been associated with an approximately $60 \%$ increase in the risk of pneumonia [3-5]. While much has been made of the lack of corresponding evidence for an increase in pneumonia related mortality, no studies have ever been adequately powered to detect differences in pneumonia-related mortality between different COPD treatments [6-8].

Studies over the past 10 years have expanded our knowledge of the effects of ICS on respiratory infections by, in addition to pneumonia, linking ICS use with an increase in active tuberculosis in observational studies [9], an increase in pneumonia in asthmatic patients [10] and even an increased frequency of upper respiratory tract infections in COPD [11]. There is little doubt therefore that ICS are powerful immunosuppressive drugs that in some cases can predispose to serious respiratory infections.

While there is evidence that ICS prescribing has declined over the subsequent decade in many countries, ICS remains a dominant treatment approach for COPD [12]. ICS use is also very common in patients with bronchiectasis and in patients with cystic fibrosis where evidence for their effectiveness is limited but where airway infection is common $[13,14]$.

Received: Aug 032017 | Accepted: Aug 042017

Conflict of interest: Disclosures can be found alongside this article at erj.ersjournals.com

Copyright @ERS 2017 
Non-tuberculous mycobacterial (NTM) infection is a serious respiratory infection that frequently complicates COPD, cystic fibrosis and bronchiectasis [15]. Rates of NTM are rising rapidly in Europe and worldwide. For example, Ringshausen et al. [16], reported an increase of $6 \%$ per year in hospital admissions for NTM disease in Germany, with COPD patients accounting for the largest proportion of cases. SHAH et al. [17] reported a 10-fold increase in the incidence of NTM from 1995 to 2012 in England, Wales and Northern Ireland. In Canada, NTM disease increased in prevalence from 29.3 per 100000 persons between 1998 and 2002 to a rate of 41.3 per 100000 population during the period 2006-2010 [18]. These represent rates of NTM in the general population. Rates in bronchiectasis have been reported from 1-9\% of patients, while the most recent data from the US Cystic Fibrosis Foundation registry found NTM-positive cultures in $12 \%$ of CF patients $[19,20]$

The reasons for rises worldwide are unclear, but may include greater awareness and testing, environmental changes promoting acquisition of NTM, or increased host susceptibility which includes the widespread use of immunosuppressive drugs in susceptible populations, such as ICS.

In this issue of the European Respiratory Journal, BRODE et al. [21] report important new data regarding the risks of ICS in patients with mixed obstructive lung disease. Using high quality linked laboratory and healthcare datasets in Canada, 417494 patients aged $\geqslant 66$ years with COPD, asthma and asthma-COPD overlap were identified. A case-control study was performed between 2001 and 2013. Cases of NTM pulmonary disease (NTM-PD) were defined using the American Thoracic Society microbiological criteria of two or more positive sputum cultures for the same species within 2 years or one bronchoscopy positive sample [21]. Each case of NTM-PD was matched to four control subjects by age, sex, year of cohort and lung disease history. Using this design, the authors sought to establish the association between use of ICS and the risk of NTM-PD in 2966 NTM-PD cases and 11851 controls [21].

The results are striking. Current ICS use nearly doubled the risk of NTM (adjusted odds ratio (aOR) 1.86, 95\% CI 1.60-2.15) with the strongest risk associated with the use of fluticasone-containing regimes (aOR 2.09, 95\% 1.80-2.43). As with pneumonia risk in COPD there appeared to be both a dose response relationship, with the highest risk for high dose fluticasone and an important influence of host susceptibility, with increased risks evident for COPD patients but less so for patients with asthma. The association remained after limiting analysis only to those individuals with treated NTM disease [21].

Case-control designs have inherent limitations and there remain risks of incomplete matching and residual confounding in such studies. Nevertheless, this is a carefully conducted and convincing epidemiological investigation that suggests a significant increase in risk of NTM infection in COPD patients receiving inhaled corticosteroids [21]. The study greatly extends a previous case control study from Denmark that included 112 NTM-PD cases and found a 29 -fold increased risk of NTM with ICS use, rising to a 47 -fold increase in those receiving the highest dose, most potent ICS preparations [22].

NTM-PD is a rare event and at an individual patient level the number needed to harm is estimated at 1775 per year. NTM risk is therefore unlikely to factor strongly into the decision of whether or not to prescribe ICS to an individual patient [21]. This study raises concerns, however, about whether widespread ICS use in patients with chronic lung disease is contributing the global rise in NTM infections. While uncommon, NTM infections are difficult to treat and associated with high healthcare costs [23, 24]. An analysis of disease burden from Germany recently showed that compared to age, sex and co-morbidity matched controls, NTM-PD patients had a near four-fold increase in mortality risk (22.4\% versus $6 \%)$ and a near four-fold increase in healthcare costs [24].

The association between ICS and NTM infection is biologically plausible. ICS is known to reduce T-cell, macrophage and neutrophil function in the lung $[25,26]$. ICS reduces total CD4 and CD8 T-cell numbers, macrophage and neutrophil numbers [26]. Recent data demonstrates reduced mucosal-associated invariant T-cells (MAIT) in blood and bronchial tissue from individuals with COPD treated with ICS and MAIT are known to be involved in host defence against mycobacteria [27]. Macrophages are also essential to host defence against NTM with TLR2 signalling, IL-12 and tumour necrosis factor- $\alpha$ (TNF- $\alpha$ ) secretion driving production of interferon- $\gamma$ in particular being of central importance. TNF- $\alpha$ secretion is suppressed by inhaled corticosteroids with fluticasone reported to be more potent than budesonide [28]. In mice, fluticasone also suppressed IL-12 production by alveolar macrophages leading to increased susceptibility to pneumococcal infection [29]. Mouse models have demonstrated that dexamethasone suppresses TNF- $\alpha$ in vivo and leads to increased mycobacterial burden in mice infected with Mycobacterium avium complex [30].

Mechanistic data in patients is lacking in respect of NTM, but is growing with regard to bacterial infection generally. ICS was associated with increased bacterial load in a cross-sectional study using quantitative PCR, while a recent study of the airway microbiome in COPD exacerbations showed that oral steroid 
TABLE 1 Current guideline recommendations for inhaled corticosteroid (ICS) use

Pulmonary disease Guideline recommendations for use of ICS

COPD [35]

Asthma [39]

Bronchiectasis [38]

Cystic fibrosis [40]
Not recommended for patients with GOLD A and B COPD (symptomatic patients without two or more exacerbations per yearl

Consider withdrawal in existing ICS users in GOLD groups A and $\mathrm{B}$

Non-preferred or second-line option for patients with GOLD C/D l patients with two or more exacerbations per year

Recommended first-line therapy

Routine use of inhaled corticosteroids is not recommended May be used in patients with co-existing asthma or COPD

Not of proven benefit for patients with cystic fibrosis Only recommended for individuals with concomitant asthma

\section{Evidence for ICS withdrawal}

Randomised controlled trials show that ICS withdrawal is safe in selected patient populations $[33,34]$

ICS withdrawal supported by GOLD strategy [35]

Not recommended

No published evidence

ICS withdrawal was not associated with increased exacerbations or deterioration in lung function [14]

COPD: chronic obstructive pulmonary disease; GOLD: Global Initiative for Obstructive Lung Disease.

treatment caused expansion of the proteobacteria phylum, the family of bacteria typically associated with pneumonia $[31,32]$.

There is now a global drive to reduce inappropriate ICS use. In COPD, several studies including the WISDOM and INSTEAD randomised controlled trials have demonstrated it is possible to withdraw inhaled corticosteroids without a corresponding increase in exacerbation frequency [33, 34]. ICS withdrawal is now recognised by the GOLD strategy as an appropriate treatment approach in selected patients, with combined long acting beta-agonists and long-acting muscarinic antagonist (LABA/LAMA) combinations recommended as first line therapy for exacerbating patients in place of ICS [35]. Peripheral blood eosinophil counts have been shown to identify a subgroup of COPD patients who respond to ICS and may also be less likely to develop ICS-related pneumonia, although the optimal cut-off to define this subgroup remains a subject of intense debate [36,37]. In bronchiectasis, there remains an absence of randomised controlled trials but the recently published European Respiratory Society guidelines recommend against the use of ICS, a recommendation that appears strengthened by the finding of an increase in NTM risk [38]. In cystic fibrosis, a previous randomised trial of 84 patients showed that withdrawal of ICS was safe with no increase in exacerbation risk and routine use of ICS is not recommended by international cystic fibrosis guidelines [14]. Table 1 summarises the current state of play for ICS use and ICS withdrawal recommendations.

Whether efforts to reduce inappropriate and ineffective ICS use will reduce the global burden of NTM disease remains to be established, but the investigation by BRODE et al. [21] adds further evidence to support the rational use of these drugs.

The risk of serious respiratory infection including pneumonia and NTM should be considered in susceptible patients when making the decision to prescribe ICS or to continue ICS in patients already on therapy. Clinicians should carefully consider the risks and benefits and be prepared to withdraw ICS in patients without a strong clinical indication.

From a research perspective, we need further studies to understand the mechanisms of increased infection susceptibility in COPD and other chronic lung diseases in order to better identify patients that should and should not receive ICS. Personalised medicine, or the pursuit of treatable traits, will require a multimodality approach including analysis of the microbiome, inflammatory profiles and clinical characteristics to identify patients in whom ICS are safe and effective, and to withdraw them in those for whom they are not.

10 years since TORCH, BRODE et al. [21] have shone a new light on the risks of ICS. The fightback against inappropriate use of ICS in chronic lung disease is gathering momentum.

\section{References}

1 Calverley PMA, Anderson JA, Celli B, et al. Salmeterol and fluticasone propionate and survival in chronic obstructive pulmonary disease. N Engl J Med 2008; 356: 775-789.

2 Crim C, Calverley PM, Anderson JA, et al. Pneumonia risk in COPD patients receiving inhaled corticosteroids alone or in combination: TORCH study results. Eur Respir J 2009; 34: 641-647. 
3 Festic E, Bansal V, Gupta E, et al. Association of inhaled corticosteroids with incident pneumonia and mortality in COPD patients; systematic review and meta-analysis. COPD 2016; 13: 312-326.

4 Vestbo J, Leather D, Diar Bakerly N, et al. Effectiveness of fluticasone furoate-vilanterol for COPD in clinica practice. N Engl J Med 2016; 375: 1253-1260.

5 Pascoe SJ, Lipson DA, Locantore N, et al. A phase III randomised controlled trial of single-dose triple therapy in COPD: the IMPACT protocol. Eur Respir J 2016; 48: 320-330.

6 Festic E, Scanlon PD. Incident pneumonia and mortality in patients with chronic obstructive pulmonary disease A double effect of inhaled corticosteroids? Am J Respir Crit Care Med 2015; 191: 141-148.

7 Sibila O, Anzueto A, Restrepo MI. The paradoxical effect on pneumonia of chronic inhaled corticosteroids. Clin Pulm Med 2013; 20.

8 Singanayagam A, Chalmers JD, Akram AR, et al. Impact of inhaled corticosteroid use on outcome in COPD patients admitted with pneumonia. Eur Respir J 2011; 38: 36-41.

9 Lee $\mathrm{CH}$, Kim K, Hyun MK, et al. Use of inhaled corticosteroids and the risk of tuberculosis. Thorax 2013; 68: 1105-1113.

10 Qian CJ, Coulombe J, Suissa S, et al. Pneumonia risk in asthma patients using inhaled corticosteroids: a quasi-cohort study. Br J Clin Pharmacol 2017; 83: 2077-2086.

11 Yang M, Chen H, Zhang Y, et al. Long-term use of inhaled corticosteroids and risk of upper respiratory tract infection in chronic obstructive pulmonary disease: a meta-analysis. Inhal Toxicol 2017; 29: 219-226.

12 Chalmers JD, Tebboth A, Gayle A, et al. Determinants of initial inhaled corticosteroid use in patients with GOLD A/B COPD: a retrospective study of UK general practice. NPJ Prim Care Respir Med 2017; $27: 43$.

13 Chalmers JD, Aliberti S, Blasi F. State of the art: management of bronchiectasis in adults. Eur Respir J 2015; 45: 1446-1462.

14 Balfour-Lynn IM, Lees B, Hall P, et al. Multicenter randomized controlled trial of withdrawal of inhaled corticosteroids in cystic fibrosis. Am J Respir Crit Care Med 2006; 173: 1356-1362.

15 Prevots DR, Loddenkemper R, Sotgiu G, et al. Nontuberculous mycobacterial pulmonary disease: an increasing burden with substantial costs. Eur Respir J 2017; 49: 1700374.

16 Ringshausen FC, Wagner D, de Roux A, et al. Prevalence of nontuberculous mycobacterial pulmonary disease, Germany, 2009-2014. Emerging Infect Dis 2016; 22: 1102-1105.

17 Shah MN, Davidson JA, Anderson LF, et al. Pulmonary Mycobacterium avium-intracellulare is the main driver of the rise in non-tuberculous mycobacteria incidence in England, Wales and Northern Ireland, 2007-2012. BMC Infect Dis 2016; 16: 195.

18 Marras TK, Mendelson D, Marchand-Austin A, et al. Pulmonary nontuberculous mycobacterial disease, Ontario, Canada, 1998-2010. Emerging Infect Dis 2013; 19: 1889-1891.

19 Aliberti S, Lonni S, Dore S, et al. Clinical phenotypes in adult patients with bronchiectasis. Eur Respir J 2016; 47: $1113-1122$.

20 Salsgiver EL, Fink AK, Knapp EA, et al. Changing epidemiology of the respiratory bacteriology of patients with cystic fibrosis. Chest 2016; 149: 390-400.

21 Brode SK, Campitelli MA, Kwong JC, et al. The risk of mycobacterial infections associated with inhaled corticosteroid use. Eur Respir J 2017; 50: 1700037.

22 Andrejak C, Nielsen R, Thomsen VO, et al. Chronic respiratory disease, inhaled corticosteroids and risk of non-tuberculous mycobacteriosis. Thorax 2013; 68: 256-262.

23 van Ingen J, Wagner D, Gallagher J, et al. Poor adherence to management guidelines in nontuberculous mycobacterial pulmonary diseases. Eur Respir J 2017; 49: 1601855.

24 Diel R, Jacob J, Lampenius N, et al. Burden of non-tuberculous mycobacterial pulmonary disease in Germany. Eur Respir J 2017; 49: 1602109.

25 Dicker AJ, Crichton ML, Pumphrey EG, et al. Neutrophil extracellular traps are associated with disease severity and microbiota diversity in patients with chronic obstructive pulmonary disease. J Allergy Clin Immunol 2017; in press [https://doi.org/10.1016/j.jaci.2017.04.022]

26 Barnes NC, Qiu YS, Pavord ID, et al. Antiinflammatory effects of salmeterol/fluticasone propionate in chronic obstructive lung disease. Am J Respir Crit Care Med 2006; 173: 736-743.

27 Hinks TS, Wallington JC, Williams AP, et al. Steroid-induced deficiency of mucosal-associated invariant T cells in the chronic obstructive pulmonary disease lung. Implications for nontypeable Haemophilus influenzae infection. Am I Respir Crit Care Med 2016; 194: 1208-1218.

28 Calverley PM. Through a glass darkly: inhaled corticosteroids, airway inflammation and COPD. Eur Respir J 2017; 49: 1602201

29 Stolberg VR, McCubbrey AL, Freeman CM, et al. Glucocorticoid-augmented efferocytosis inhibits pulmonary pneumococcal clearance in mice by reducing alveolar macrophage bacteriocidal function. J Immunol 2015; 195: 174-184.

30 Bala S, Hastings KL, Kazempour K, et al. Inhibition of tumour necrosis factor alpha alters resistance to Mycobacterium avium complex infection in mice. Antimicrob Agents Chemother 1998; 42: 2336-2341.

31 Garcha DS, Thurston SJ, Patel AR, et al. Changes in prevalence and load of airway bacteria using quantitative PCR in stable and exacerbated COPD. Thorax 2012; 67: 1075-1080.

32 Wang Z, Bafadhel M, Haldar K, et al. Lung microbiome dynamics in COPD exacerbations. Eur Respir J 2016; 47: 1082-1092.

33 Magnussen $\mathrm{H}$, Tetzlaff $\mathrm{K}$, Bateman ED, et al. Lung function changes over time following withdrawal of inhaled corticosteroids in patients with severe COPD. Eur Respir J 2016; 47: 651-654.

34 Rossi A, van der Molen T, del Olmo R, et al. INSTEAD: a randomised switch trial of indacaterol versus salmeterol/fluticasone in moderate COPD. Eur Respir J 2014; 44: 1548-1556.

35 Vogelmeier CF, Criner GJ, Martinez FJ, et al. Global Strategy for the Diagnosis, Management, and Prevention of ChronicObstructive Lung Disease 2017 Report: GOLD Executive Summary. Eur Respir J 2017; 49: 1700214.

36 Agusti A, Bel E, Thomas M, et al. Treatable traits: toward precision medicine of chronic airway diseases. Eur Respir J 2016; 47: 410-419.

37 Pavord ID, Lettis S, Anzueto A, et al. Blood eosinophil count and pneumonia risk in patients with chronic obstructive pulmonary disease: a patient-level meta-analysis. Lancet Respir Med 2016; 4: 731-741. 
38 Polverino E, Goeminnes PC, McDonnell M, et al. European Respiratory Society guidelines for the management of adult bronchiectasis. Eur Respir J 2017; 50: 1700629.

39 Reddel HK, Bateman ED, Becker A, et al. A summary of the new GINA strategy: a roadmap to asthma control. Eur Respir J 2015; 46: 622-639.

40 Smyth AR, Bell SC, Bojcin S, et al. European Cystic Fibrosis Society standards of care; best practice guidelines. J Cyst Fibros 2014; 13: Suppl. 1, S23-S42. 